\title{
Quasi-LPV Unknown Input Observer with Nonlinear Outputs: Application to Motorcycles
}

\author{
L. Nehaoua, M. Fouka and H. Arioui
}

\begin{abstract}
The purpose of the present work is the reconstruction of motorcycle lateral dynamics. The main idea is to estimate pertinent states and unknown inputs (rider action) with respect to nonlinear outputs due to motion transformation frames (inertial sensors are away from the local frame). To overcome this issue, we propose a new Unknown Input Observers with variable output matrix. In this paper, we take into account the ground truth measurements provided in the body-fixed frame, parametric uncertainties as well as sensors noise. This step leads to a nonlinear parameter-dependent output equation with unmeasured premise variables in the observer design. The observer synthesis is specified in term of convergence and stability study by considering a quadratic Lyapunov function associated with the Input To State Stability (ISS) property. Sufficient conditions are agreed in terms of Linear Matrix Inequalities (LMIs). Finally, the performances, usefulness and robustness of the proposed approach are assessed throughout an electric Scooter under urban riding scenario.
\end{abstract}

Index Terms-Nonlinear Output, Quasi-LPV Observer, ISS Stability, Motorcycle Lateral Dynamics.

\section{INTRODUCTION}

Nowadays, vehicles are increasingly sophisticated and equipped with advanced driver assistance systems (ADAS). The objective of these assistance systems is to provide vehicles, the environment and the infrastructure with intelligence and autonomy. In fact, autonomous driving and road safety have become real challenges, therefore ADAS development is attracting great interest, particularly around vehicle localization, autonomous guidance, obstacle avoidance, pedestrian detection, stability control, etc. Most of them are dedicated to improve safety by warning the driver of dangerous situations and sometimes by acting on the dynamics of the vehicle. Unfortunately, we are far from this excitement from the bike side. Indeed, the powered motorized two-wheeled vehicle (PWTv) market being less expensive, it is difficult to cover instrumentation and Research \& Development costs with an attractive selling price. In addition, most of these assistance systems are based on mathematical models of the vehicle, and motorcycles are much more complex to model and highly non-linear than four-wheeled vehicles. The delay in the development of advanced assistance systems is not without consequence since accidentology of motorcyclists is really alarming worldwide [1].

Today some safety systems exist on motorcycle market: Braking System (ABS), Traction Control System (TCS), Motorcycle Stability Control (MSC), etc. But the majority of

All the authors are with the Informatics, Integrative Bio-Informatics and Complex Systems Laboratory (IBISC), Evry Val d'Essonne (UEVE)- ParisSaclay University, 40, rue de Pelvoux, 91020 Evry Courcouronne Cedex, France. hichem. ariouiduniv-evry. fr them are set on premium motorcycles class which represent a little proportion of the motorcycle park. Estimation techniques are main outfits to make easier the development of ADAS and hence reducing the cost. Lot of recent works deal with motorcycle dynamic state estimation [2], [3], [4] but few of them perform experimental investigations to validate the results. In [5] and [6] experimental tests are performed but only lateral dynamics estimation is considered. In [7] authors proposed a more complete experimental investigation to validate estimated states with extended Kalman filter but only constant forward speed case is considered. This issue was addressed in [8]. Unfortunately, all these works consider a constant output matrix which is a strong assumption. Because of the distance between the inertial measurement (near to the center of gravity) and body fixed frame. This induces significant errors in certain riding conditions (cornering, high speed, etc.). We introduced recently an interesting extension to our previous works on the estimation of the motorcycle's lateral and steering dynamics with nonlinear outputs [6] where we present here some updates as well as an experimental application to motorcycles.

Presently, the Inertial Measurement Unit (IMU) is the best motion sensor used in automotive applications. IMUs provide a measurement in the six Degrees of Freedom (DoF) of the vehicle motion. However, these measurements should be transformed to the vehicle body frame. Otherwise, the IMU mounting should be included to reformulate the dynamics model output in order to be coherent with sensor measurements. This reformulation could enhance the estimation algorithm and allows avoiding significant errors in the PTWv's motion interpretation with respect to the car vehicle where the roll angle is neglected.

The main contribution of this paper is the robustness study towards the parametric uncertainties and sensors noise and, on the other hand, the experimental validation of the proposed works in [6] by designing a new unknown input observer (UIO) with variable output matrix. The observer design is based on a simple two bodies model of the electric scooter which allows to get very good estimation time performance. Let us remind that the considered UIO is able to simultaneously estimate every lateral dynamic states and rider action without any forward speed limitation.

This paper is structured as follows: Section II introduce dynamic modeling and misalignment problem. Section III explains how the proposed robust observer is derived. Section IV discusses the results of the experimental tests. Finally, section $\mathrm{V}$ presents concluding remarks and discussions. 


\section{Dynamic Model Description}

\section{A. Lateral Dynamics Description}

The lateral dynamics of the PTWv (Fig. 1) is considered as presented in Sharp's 71 model [9]. This model captures the lateral displacement $v_{y}$, the yaw motion $\psi$, the roll motion $\phi$ of the main vehicle's body $G_{r}$ and the steering motion $\delta$ of the front body $G_{f}$. These $4 \mathrm{DoF}$ are combined with the relaxation behavior of the nonlinear front and rear tire forces $F_{y f}$ and $F_{y r}$. By choosing the state vector $x(t)=\left[\begin{array}{llllllll}\phi & \delta & v_{y} & \dot{\psi} & \dot{\phi} & \dot{\delta} & F_{y f} & F_{y r}\end{array}\right]^{T}$, the PTWv dynamics state-space representation is:

$$
\bar{E} \dot{x}=\bar{A}\left(v_{x}(t)\right) x(t)+\bar{B} \tau(t)
$$

where $\tau$ is the unknown rider's torque and $v_{x}(t)$ is the timevarying forward speed. Matrices $\bar{E}=\left[e_{i j}\right], \bar{A}\left(v_{x}\right)=\left[a_{i j}\right]$ and $\bar{B}$ and their parameters are given in appendix [6].

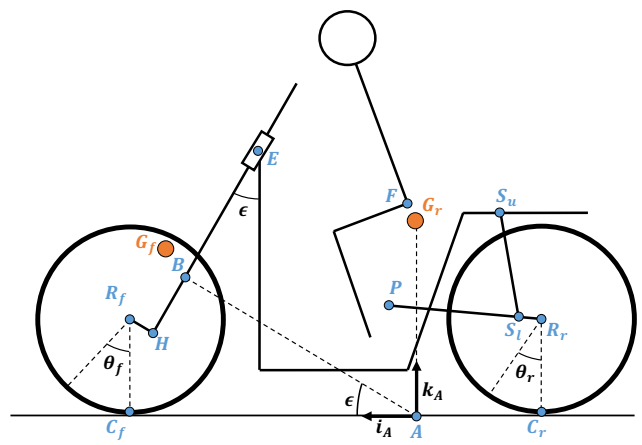

Fig. 1. The geometry of the two-body model of the PTWV.

\section{B. Motorcycle Instrumentation and Sensor Alignment}

The IMU sensor is usually mounted at point $G_{m}$ near to the center of gravity of the main motorcycle's body, $G_{r}$. It provides the three body-fixed linear accelerations $a_{x b f}, a_{y b f}$ and $a_{z b f}$ and the three angular rates $\dot{\psi}_{b f}, \dot{\phi}_{b f}$ and $\dot{\theta}_{b f}$. All these measurements are expressed in the IMU body-fixed frame as illustrated in Fig. 2.

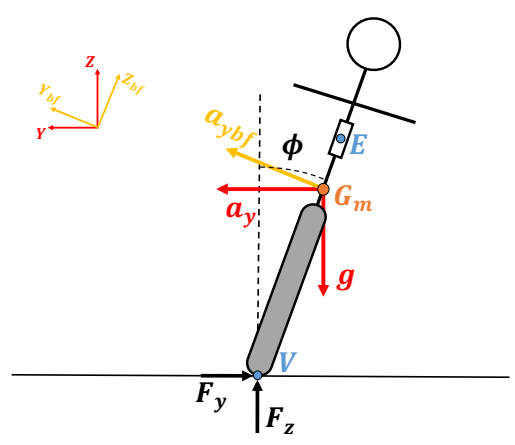

Fig. 2. The PTWv accelerations expressed in the IMU reference frame.

In almost study cases, the dynamics model's outputs expressed in the vehicle reference frame $\left(A, \vec{i}_{A}, \vec{j}_{A}, \vec{k}_{A}\right)$, and the IMU measurements expressed in the IMU reference frame
$\left(G_{m}, \vec{i}_{b f}, \vec{j}_{b f}, \vec{k}_{b f}\right)$ are supposed to be the same. If this assumption is relatively true for cars, it is not coherent with the PTWv motion where the roll angle can reach $45^{\circ}$. In that case, this assumption considerably affects the system's observability.

Let's denote $\cos (\beta), \sin (\beta), \tan (\beta)$ and $\operatorname{sinc}(\beta)$ with their abbreviations $\mathrm{c}_{\beta}, \mathrm{s}_{\beta}, \mathrm{t}_{\beta}$ and $\mathrm{sc}_{\beta}$, where $\operatorname{sinc}(\beta)=\frac{\sin (\beta)}{\beta}$. Since the IMU box is not aligned with the vehicle reference frame, then the accelerations in the $Y$ and $Z$ axis in the body-fixed frame can be approximated by:

$$
\begin{aligned}
& a_{y b f}=a_{y} c_{\phi}-g s_{\phi} \\
& a_{z b f}=-a_{y} s_{\phi}-g c_{\phi}
\end{aligned}
$$

In addition, in cornering steady state, the lateral acceleration in the vehicle reference frame can be computed from the tire forces as $M a_{y}=F_{y f}+F_{y r}$. Thus, acceleration $a_{y b f}$ becomes:

$$
a_{y b f}=\frac{c_{\phi}}{M}\left(F_{y f}+F_{y r}\right)-g s_{\phi}
$$

On the other hand, the vehicle orientation is described by Euler angles: roll $(\phi)$, pitch $(\theta)$ and yaw $(\psi)$ which are related to the IMU body-fixed angular rates by [10]:

$$
\left\{\begin{aligned}
\dot{\phi} & =\dot{\phi}_{b f}+t_{\theta} s_{\phi} \dot{\theta}_{b f}+c_{\phi} t_{\theta} \dot{\psi}_{b f} \\
\dot{\theta} & =c_{\phi} \dot{\theta}_{b f}-s_{\phi} \dot{\psi}_{b f} \\
\dot{\psi} & =s_{\phi} / c_{\theta} \dot{\theta}_{b f}+c_{\phi} / c_{\theta} \dot{\psi}_{b f}
\end{aligned}\right.
$$

By some algebraic manipulations, the body-fixed angular rates are expressed as follows:

$$
\dot{\theta}_{b f}=\dot{\psi} s_{\phi}, \quad \dot{\psi}_{b f}=\dot{\psi} t_{\phi} s_{\phi}, \quad \dot{\phi}_{b f}=\dot{\phi}
$$

Hence, the IMU sensor outputs are established and can be related to the state vector $x(t)$ by:

$$
y(t)=\left[\delta(t), \dot{\psi}_{b f}(t), \dot{\phi}_{b f}(t), \dot{\delta}(t), a_{y_{b f}}(t)\right]^{T}=C(x(t)) x(t)
$$

where the output matrix $C(x(t))$ is expressed by:

$$
C(x(t))=\left[\begin{array}{cccccccc}
0 & 1 & 0 & 0 & 0 & 0 & 0 & 0 \\
0 & 0 & 0 & t_{\phi} s_{\phi} & 0 & 0 & 0 & 0 \\
0 & 0 & 0 & 0 & 1 & 0 & 0 & 0 \\
0 & 0 & 0 & 0 & 0 & 1 & 0 & 0 \\
-g . s c_{\phi} & 0 & 0 & 0 & 0 & 0 & \frac{c_{\phi}}{M} & \frac{c_{\phi}}{M}
\end{array}\right]
$$

Since the matrix $\bar{E}$ is non-singular, the model given by (1) can be written in a quasi linear parameter varying (LPV) form as:

$$
\left\{\begin{array}{l}
\dot{x}(t)=A\left(v_{x}(t)\right) x(t)+B \tau(t) \\
y=C(x(t)) x(t)
\end{array}\right.
$$

where: $A\left(v_{x}\right)=\bar{E}^{-1} \bar{A}\left(v_{x}\right)$ and $B=\bar{E}^{-1} \bar{B}$.

\section{TS Structure of the Lateral Dynamics Model}

In this section, by using the sector nonlinearity approach, the system dynamics (7) with its $q$ nonlinearities is exactly rewritten as weighted $r$ linear sub-models [11]:

$$
\left\{\begin{array}{l}
\dot{x}(t)=A_{\mu} x(t)+B \tau(t) \\
y(t)=C_{\mu} x(t)
\end{array}\right.
$$

where $A_{\mu}=\sum_{i=1}^{r} \mu_{i}(v) A_{i}$ and $C_{\mu}=\sum_{i=1}^{r} \mu_{i}(v) C_{i}$ and, matrices $A_{i}, B$, and $C_{i}$ are constant. Also weighting functions satisfy $0 \leq \mu_{i}(v) \leq 1$ and $\sum_{i=1}^{r} \mu_{i}(v)=1$ with $r=2^{q}$. 
The vector $v(t)=\left[\begin{array}{llll}v_{x} & \operatorname{sc}_{\phi} & \mathrm{t}_{\phi} \mathrm{s}_{\phi} & \mathrm{c}_{\phi}\end{array}\right]^{T}$ is the vector of the premise variables. The longitudinal velocity $v_{x} \in$ $\left[v_{\min }, v_{\max }\right]$ is measurable and bounded. Also, the roll angle is bounded and belongs to $\left[\phi_{\min }, \phi_{\max }\right]$ for a stable motorcycle.

\section{OBSERVER DESIGN}

The aim of this part is to design a nonlinear unknown input observer (UIO) for Takagi-Sugeno (TS) fuzzy systems under parameters uncertainties $\Delta A_{\mu}$ and sensor noise $n(t)$ as :

$$
\left\{\begin{array}{l}
\dot{x}(t)=\left(A_{\mu}+\Delta A_{\mu}\right) x(t)+B \tau(t) \\
y(t)=C_{\mu} x(t)+D n(t)
\end{array}\right.
$$

$D$ is a constant matrix. In this context, the following nonrestrictive assumptions are considered:

(i) The state $x(t)$ and the steering torque $\tau(t)$ are bounded i.e., stable or stabilized motorcycle motion;

(ii) The pairs $\left(A_{i}, C_{j}\right)$ are observable or detectable;

(iii) The matching condition holds $\operatorname{rank}\left(C_{i} B\right)=\operatorname{rank}(B)$;

(iv) The sensor noise and its derivative are bounded

$$
\|n(t)\|_{\infty}<\infty \text { and }\left\|\frac{d n(t)}{d t}\right\|_{\infty}<\infty
$$

(v) The model uncertainties are bounded $\left\|\Delta A_{\mu}\right\|_{\infty}<\infty$.

Unlike other UIO approaches, we focus here on using quasiLPV UIO with state dependent output matrix and unmeasurable premise variables to estimate the system's states and unknown inputs.

\section{A. State estimation}

In the present paper, we consider the nonlinear observer, in TS form, given by:

$$
\left\{\begin{array}{l}
\dot{z}(t)=N_{\hat{\mu}} z(t)+L_{\hat{\mu}} y(t) \\
\hat{x}(t)=z(t)-M_{\hat{\mu}} y(t)
\end{array}\right.
$$

where, $\hat{x}(t)$ is the estimated state and $y(t)$ is the output vector. $\hat{\mu}$ are the estimated weighting functions. The observer's matrices $N_{\hat{\mu}} \in \mathbb{R}^{n \times n}, L_{\hat{\mu}} \in \mathbb{R}^{n \times n_{y}}, M_{\hat{\mu}} \in \mathbb{R}^{n \times n_{y}}$ are parameter varying and have the same quasi-LPV form as the matrix $A_{\mu}$.

$$
N_{\hat{\mu}}=\sum_{i=1}^{r} \hat{\mu}_{i}(v) N_{i}, \quad L_{\hat{\mu}}=\sum_{i=1}^{r} \hat{\mu}_{i}(v) L_{i}, M_{\hat{\mu}}=\sum_{i=1}^{r} \hat{\mu}_{i}(v) M_{i}
$$

The observer design procedure aims to determine the aforementioned observer's matrices. According to (10), the state estimation error $e(t)=x(t)-\hat{x}(t)$ can be written as:

$$
\begin{aligned}
e(t) & =x(t)-\hat{x}(t) \\
& =x(t)-z(t)+M_{\hat{\mu}} C_{\mu} x(t)+M_{\hat{\mu}} D n(t) \\
& =P_{\hat{\mu}} x(t)-z(t)+\zeta(t)
\end{aligned}
$$

where: $P_{\hat{\mu}}=I-M_{\hat{\mu}} C_{\hat{\mu}}$ and $\zeta(t)=M_{\hat{\mu}}\left(C_{\hat{\mu}}-C_{\mu}\right) x(t)-$ $M_{\hat{\mu}} D n(t)$, which one can minimize its effect on the estimation error. By differentiating (12) and by using equations (9) and (10), the error dynamics is:

$$
\begin{aligned}
\dot{e}(t) & =\dot{P}_{\hat{\mu}} x(t)+P_{\hat{\mu}} \dot{x}(t)-\dot{z}(t)+\dot{\zeta}(t) \\
& =N_{\hat{\mu}} e(t)+\left(\dot{P}_{\hat{\mu}}+P_{\hat{\mu}} A_{\hat{\mu}}-L_{\hat{\mu}} C_{\hat{\mu}}-N_{\hat{\mu}} P_{\hat{\mu}}\right) x(t)+P_{\hat{\mu}} B \tau(t)+d(t)
\end{aligned}
$$

where $d(t)=P_{\hat{\mu}}\left(A_{\mu}-A_{\hat{\mu}}+\Delta A_{\mu}\right) x(t)+L_{\hat{\mu}}\left(C_{\hat{\mu}}-C_{\mu}\right) x(t)-$ $N_{\hat{\mu}} \zeta(t)+\dot{\zeta}(t)$. According to assumptions (III.i), (III.iv) and
(III.v), the state vector $x(t)$ is stable and, since the weighting functions $\mu_{i}$ are positive and convex, then $\zeta(t)$ and $\dot{\zeta}(t)$ are bounded. This will fit the general practical case of a stable PTWv.

\section{B. Observer's convergence study}

Once the system and the observer are written in TS form, the UIO design reduces to the stability analysis of the estimation error dynamics. This is achieved based on Lyapunov theory and the ISS property which yields to an LMI optimization problem. If the observer's matrices can be computed such:

$$
\begin{aligned}
& \dot{P}_{\hat{\mu}}+P_{\hat{\mu}} A_{\hat{\mu}}-N_{\hat{\mu}} P_{\hat{\mu}}-L_{\hat{\mu}} C_{\hat{\mu}}=0 \\
& P_{\hat{\mu}} B=0
\end{aligned}
$$

Then, the error dynamics in (13) is reduced to [12]:

$$
\dot{e}(t)=N_{\hat{\mu}} e(t)+d(t)
$$

If $d(t)$ is vanishing with time, i.e., when $t \rightarrow \infty$, then convergence of the estimation error $e(t)$ is asymptotic $\left(N_{\hat{\mu}}\right.$ is Hurwitz). Otherwise, ISS performances can be achieved for the estimation error $e(t)$ as it will be stated in theorem 1 .

The second condition (15) is called decoupling condition. From (12), we know that $P_{\hat{\mu}}=I_{n}+M_{\hat{\mu}} C_{\hat{\mu}}$. Then, the condition (15) holds as stated by assumption (III.iii). This leads to:

$$
P_{\hat{\mu}} B=0 \Rightarrow M_{\hat{\mu}}=-B\left(C_{\hat{\mu}} B\right)^{\dagger}
$$

In the same way, by differentiating (15), we get $\dot{M}_{\hat{\mu}}$ :

$$
\dot{P}_{\hat{\mu}} B=0 \Rightarrow\left(\dot{M}_{\hat{\mu}} C_{\hat{\mu}}+M_{\hat{\mu}} \dot{C}_{\hat{\mu}}\right) B=0
$$

Then:

$$
\dot{M}_{\hat{\mu}}=-M_{\hat{\mu}} \dot{C}_{\hat{\mu}} B\left(C_{\hat{\mu}} B\right)^{\dagger}=M_{\hat{\mu}} \dot{C}_{\hat{\mu}} M_{\hat{\mu}}
$$

By using $P_{\hat{\mu}}$, the first condition (14) can be expressed as:

$$
N_{\hat{\mu}}=\Gamma_{\hat{\mu}}-K_{\hat{\mu}} C_{\hat{\mu}}
$$

where $\Gamma_{\hat{\mu}}=\dot{P}_{\hat{\mu}}+P_{\hat{\mu}} A_{\hat{\mu}}$ and $K_{\hat{\mu}}=N_{\hat{\mu}} M_{\hat{\mu}}+L_{\hat{\mu}}$. Consequently, the error dynamics becomes:

$$
\dot{e}(t)=\left(\Gamma_{\hat{\mu}}-K_{\hat{\mu}} C_{\hat{\mu}}\right) e(t)+d(t)
$$

\section{Stability Analysis}

The observer stability is studied by using the Lyapunov theory to prove the exponential stability with respect to the disturbance term $d(t)$ and, the estimated membership functions $\hat{\mu}$. In this case, the ISS property will be formulated as an optimization problem under LMI conditions.

Definition 1: [13] The state estimation error dynamics verifies the ISS if there exists a $\mathscr{K} \mathscr{L}$ function $f_{1}: \mathbb{R}^{n} \times \mathbb{R} \longrightarrow \mathbb{R}$, a $\mathscr{K}$ function $f_{2}: \mathbb{R} \longrightarrow \mathbb{R}$ such that for each input $d(t)$ satisfying $\|d\|_{\infty}<\infty$ and each initial conditions $e(0)$, the trajectory of the error associated to $e(0)$ and $d(t)$ satisfies

$$
\|e(t)\|_{2} \leq f_{1}(\|e(0)\|, t)+f_{2}\left(\|d(t)\|_{\infty}\right)
$$

Definition 2 (The Schur's complement ): [14] Given the matrices $Q, S$ and $R$, with appropriate dimensions, where 
$S=S^{T}$ and $R=R^{T}$, then the following equivalence holds:

$$
\left[\begin{array}{cc}
S & Q \\
Q^{T} & R
\end{array}\right]>0 \Leftrightarrow\left\{\begin{array}{l}
R>0 \\
S-Q R^{-1} Q^{T}>0
\end{array}\right.
$$

Theorem 1: Provided the polytopic system (9) under stated assumptions. Given the varying parameters dependent matrices $M_{\hat{\mu}}$ and $\Gamma_{\hat{\mu}}$ satisfying (17) and (19) and a positive scalar $\gamma$. If there exist a symmetric positive definite matrix $Q$, matrices $K_{i}$, and a positive scalar $\eta$ solutions of the following LMI optimization problem:

$$
\min _{R, Q, \eta} \eta
$$

such that and, for $i=1, \cdots, r$ :

$$
\begin{gathered}
{\left[\begin{array}{cc}
\Gamma_{i}^{T} Q+Q \Gamma_{i}-C_{i}^{T} R_{i}^{T}-R_{i} C_{i}+\alpha Q & Q \\
Q & -\gamma I
\end{array}\right]<0} \\
\left(\begin{array}{cc}
\alpha \eta I & Q \\
Q & \alpha \eta I
\end{array}\right)>0, \quad Q \geq I
\end{gathered}
$$

then the state estimation error $e(t)=\hat{x}(t)-x(t)$ has an Input To State Stability property and converges to an origin-centered ball region.

Proof 1:

Let's consider the quadratic Lyapunov function:

$$
V(t)=e(t)^{T} Q e(t), \quad Q=Q^{T}>0
$$

Its time derivative along the trajectory of the state estimation error is given by :

$$
\begin{aligned}
\dot{V}(t) & =e(t)^{T} \sum_{i=1}^{r}\left(\Gamma_{i}^{T} Q+Q \Gamma_{i}-C_{i}^{T} K_{i}^{T} Q-Q K_{i} C_{i}\right) e(t) \\
& +d(t)^{T} Q e(t)+e(t)^{T} Q d(t)
\end{aligned}
$$

By adding and subtracting the term $\alpha e(t)^{T} Q e(t)+\gamma d^{T}(t) d(t)$, with $\alpha$ and $\gamma$ a positive scalars, the inequality (26) can be rewritten as following:

$\dot{V}(t) \leq\left[\begin{array}{l}e(t) \\ d(t)\end{array}\right]^{T} \Pi_{\hat{\mu}}\left[\begin{array}{l}e(t) \\ d(t)\end{array}\right]-\alpha e(t)^{T} Q e(t)+\gamma d(t)^{T} d(t)$

where $\Pi_{\hat{\mu}}=\sum_{i=1}^{r} \hat{\mu}_{i}(v) \Pi_{i}$ and:

$$
\Pi_{i}=\left[\begin{array}{cc}
\Gamma_{i}^{T} Q+Q \Gamma_{i}-C_{i}^{T} R_{i}^{T}-R_{i} C_{i}+\alpha Q & Q \\
Q & -\gamma I
\end{array}\right]
$$

with $R_{i}=Q K_{i}$. Now, if $\Pi<0$, then the time derivative of the Lyapunov function (27) can be bounded as follows:

$$
\dot{V}(t) \leq-\alpha V(t)+\gamma d(t)^{T} d(t)
$$

By integrating (29), we get:

$$
\begin{aligned}
V(t) & \leq V(0) e^{-\alpha t}+\gamma \int_{0}^{t} e^{-\alpha(t-s)}\|d(s)\|_{2}^{2} d s \\
& \leq V(0) e^{-\alpha t}+\frac{\gamma}{\alpha}\|d(t)\|_{\infty}^{2}
\end{aligned}
$$

Knowing that $V(t)$ is a Lyapunov function, it can be bounded by $\lambda_{\min }\|e(t)\|_{2}^{2}$ and $\lambda_{\max }\|e(t)\|_{2}^{2}$, where $\lambda_{\min }$ and $\lambda_{\max }$ are the min and max eigenvalues of the matrix $Q$, hence:

$$
\|e(t)\|_{2}^{2} \leq \frac{\lambda_{\max }}{\lambda_{\min }}\left(\|e(0)\|_{2}^{2} e^{-\alpha t}+\frac{\gamma}{\alpha}\|d(t)\|_{\infty}^{2}\right)
$$

By using the square root on (31), we obtain:

$$
\|e(t)\|_{2} \leq \sqrt{\frac{\lambda_{\max }}{\lambda_{\min }}}\|e(0)\|_{2} e^{-\frac{\alpha}{2} t}+\sqrt{\frac{\lambda_{\max }}{\lambda_{\min }}} \sqrt{\frac{\gamma}{\alpha}}\|d(t)\|_{\infty}
$$

where:

$$
\begin{aligned}
\|d(t)\|_{\infty} \leq & \left\|P_{\hat{\mu}}\right\|_{\infty}\|x(t)\|_{\infty} \\
& +\left\|P_{\hat{\mu}} \Delta A_{\mu} x(t)\right\|_{\infty}+\left\|N_{\hat{\mu}} \zeta(t)\right\|_{\infty}+\|\dot{\zeta}(t)\|_{\infty}
\end{aligned}
$$

From the boundedness of $\zeta(t)$ and $\dot{\zeta}(t)$ and, thanks to definition (1), it is shown that the error dynamics (20) is stable and verifies the ISS property. The states estimation converges to a ball region with an ISS gain which can be minimized in order to achieve a more accurate state estimation of the motorcycle lateral motion. Since $\lambda_{\min }$ can be imposed to be greater than one and $\gamma$ can also be fixed, therefore, minimizing the ISS gain is equivalent to minimizing a positive scalar $\eta$ such that:

$$
\sqrt{\frac{\lambda_{\max }}{\alpha}} \leq \sqrt{\eta} \Leftrightarrow(\alpha \eta)^{2} I-Q^{T} Q>0
$$

By applying the Schur's complement given in definition (2), the inequality (33) can be written as an LMI constraint as:

$$
(\alpha \eta)^{2} I-Q^{T} Q>0 \Leftrightarrow\left[\begin{array}{cc}
\alpha \eta I & Q \\
Q^{T} & \alpha \eta I
\end{array}\right]
$$

The observer design procedure is summarized in the following steps:

1) Compute matrix $M_{\hat{\mu}}$ from (17) and matrix $P_{\hat{\mu}}$ from (12),

2) Solve the LMIs in (23) and (24),

3) Compute the observer gains $K_{i}$ and deduce $N_{i}$ and $L_{i}$ from (35),

4) Use (11) to determine the observer's matrices.

$$
\begin{aligned}
K_{i} & =Q_{i}^{-1} R_{i} \\
N_{i} & =\Gamma_{i}-K_{i} C_{i} \\
L_{i} & =K_{i}-N_{i} M_{i}
\end{aligned}
$$

\section{EXPERIMENTAL RESULTS}

\section{A. Experimental Steup}

A PTWv Peugeot Scoot'elec is instrumented in order to perform the tests (Fig. 3). It is equipped with an electric powertrain developing a maximum power around $3 \mathrm{~kW}$ allowing a maximum speed of $45 \mathrm{~km} / \mathrm{h}$. Three blocks of NiCad batteries with, for each one, a capacity of 100Ah for $6 \mathrm{~V}$ voltage feed the motor and inevitably lead to a consequent weight of 115 $\mathrm{kg}$ without rider.

In order to achieve the several designs and dynamic estimation, we have chosen an embedded computer manufactured by Neousys Technology. According to its compact size the model NUVO-3005EB is ideal for installation under a seat. It offers several features like: High performance CPU (Intel Core i73610QM), PCIe Expansion Slot, Wi-Fi, 3G and GPS. 


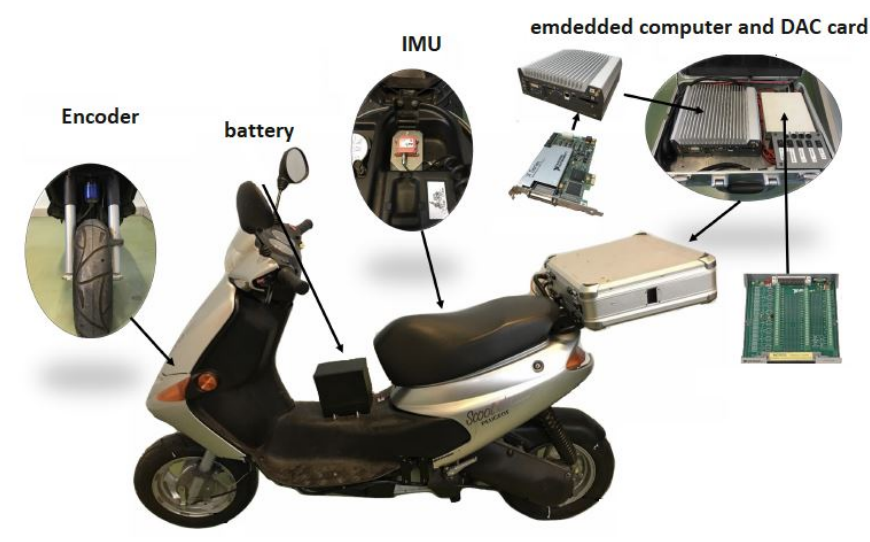

Fig. 3. Instrumented Scooter at the IBISC Lab.

The Inertial Measurement Unit (IMU) is a IG-500A manufactured by SBG Systems, Fig. 3. It can work on angular movements of $360^{\circ}$ on the 3 axes and offers orientation matrix either on Cartesian or Euler angles. The IMU is placed as close as possible to the gravity center.

Several methods are possible to instrument the steering mechanism. We made the choice of an absolute encoder to measure the handlebar angle $\delta$, directly installed on the steering column and, gives the steering angle without any transformation or ratio.

\section{B. Results Analysis}

The expected urban scenario is considered with a varying longitudinal velocity from $5 \mathrm{Km} / \mathrm{h}$ to $40 \mathrm{Km} / \mathrm{h}$, as depicted in Fig. 4.
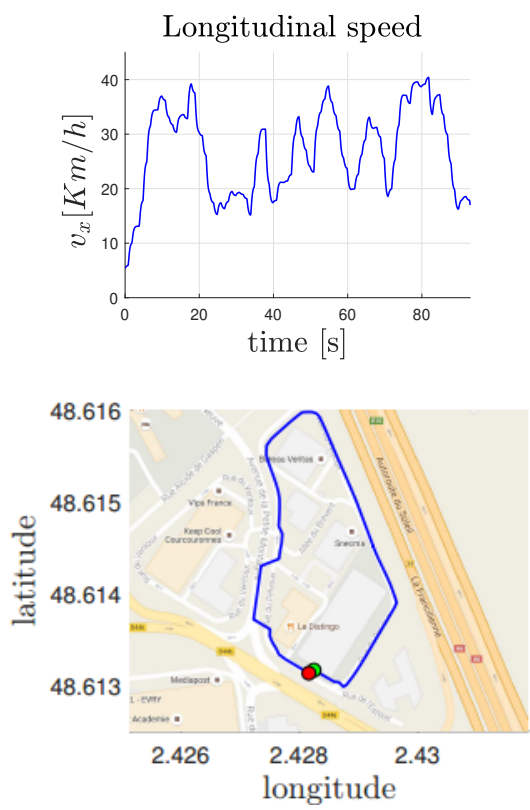

Fig. 4. Longitudinal velocity and Vehicle trajectory

The observer estimates the lateral dynamics (mainly lateral velocity $v_{y}$, roll angle $\phi$ and lateral forces $\left.F_{y r} / F_{y f}\right)$ using the measured states given by the inertial unit and the steering encoder, depicted in Fig. 5. Also, the estimation error is plotted to illustrate the observer performance and the boundedness of the estimation errors.

Fig. 6 shows the estimation of the unmeasurable states, namely the front and rear cornering forces and the lateral speed. Then, by an algebraic inversion from (36), one can reconstruct the rider torque $\hat{\tau}(t)$ applied on the handlebar:

$$
\hat{\tau}(t)=\left(C_{\hat{\mu}} B\right)^{-1}\left(\dot{y}(t)-\left(\dot{C}_{\hat{\mu}}+C_{\hat{\mu}} A_{\hat{\mu}}\right) \hat{x}(t)\right)
$$

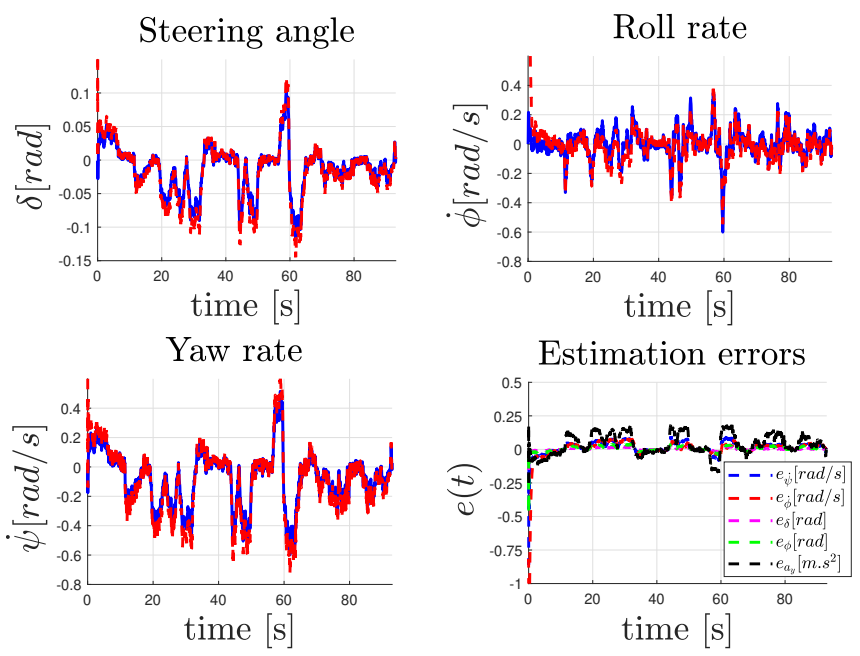

Fig. 5. States estimation (dashed red) compared to actual measurement (blue).

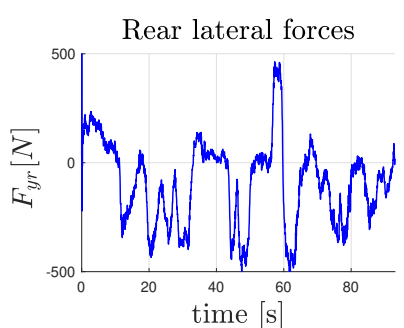

Lateral speed

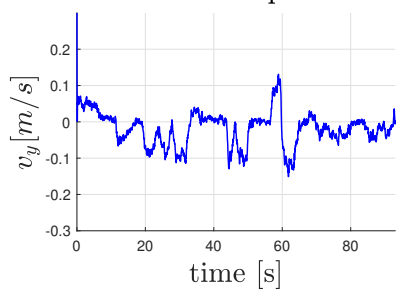

Front lateral forces

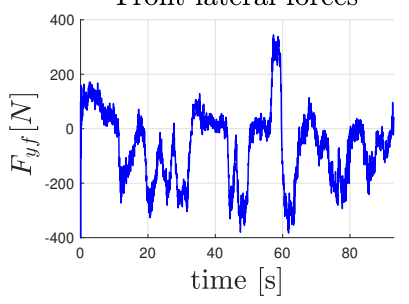

Steering torque

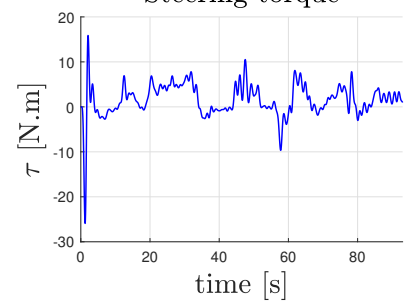

Fig. 6. Unmeasurable states estimation of Scooter and torque estimation from model inversion.

Since the actual state $v_{y}$ and $F_{y f}, F_{y r}$ are unknown, the state estimation cannot be checked directly. One can use the estimates $\hat{v}_{y}$ and $\hat{F}_{y f}, \hat{F}_{y r}$ to reconstruct the lateral acceleration $a_{y}$ which is the sum of the estimated lateral forces (37). Also, the roll measurement from the IMU can be used to check the estimated one $\hat{\phi}$ (see Fig. 7).

$$
\hat{a}_{y}=\frac{\left(\hat{F}_{y f}+\hat{F}_{y r}\right)}{M}
$$


The ability of the designed observer to well recover simultaneously the motorcycle dynamics and the unknown inputs can be highlighted with an acceptable convergence to the actual value. This scenario endorses that this observer is able to estimate the lateral dynamics and the rider action even for real riding situations. Nevertheless, there are some differences at the peak area of the estimated states due to modeling uncertainties between the Scooter and the estimated model. It should be noted that while the lateral model estimation algorithm was developed using two bodies motorcycle model (4 DoF), the data are being collected using real motorcycle dynamics.

Also, let us remind that the observer is based on ISS property. Finally, this also explains the non asymptotic estimation errors convergence.
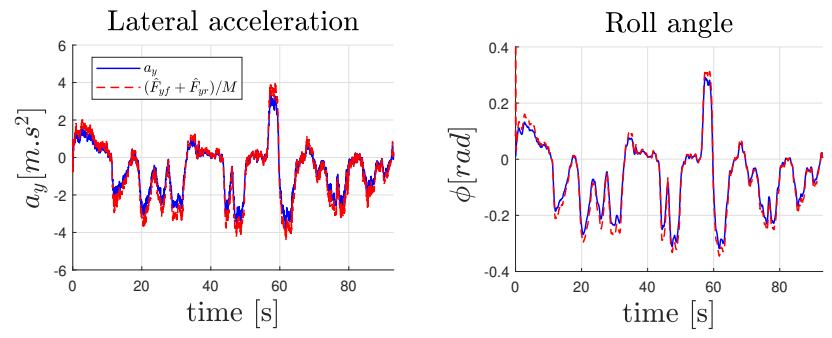

Fig. 7. Validation $: v_{y}, F_{y f}, F_{y r}$ from the lateral acceleration and $\phi$ from IMU roll angle measurement.

\section{CONCLUSION}

In this paper, the problem of unknown input observer design for quasi-LPV motorcycle system with IMU data calibration is investigated. This class of systems addressed here is timevarying parameters dependent on unmeasurable states. The main contributions of the proposed approach is to reconsider the classical unknown input observer synthesis nonlinear output. The resulting observer allows to estimate the lateral dynamic states and to reconstruct the unknown rider steering torque applied on the motorcycle handlebar. In the other hand, the motorcycle dynamics is modeled in the IMU reference frame where the system's outputs are measured directly by the Inertial Measurement Unit without additional reference frame transformation. Then, by using input to state stability property, the convergence of the states estimation is proved and formulated as an optimization problem under LMI conditions. Finally, the observer is validated throughout experimental data to reconstruct the relevant unmeasurable states and unknown input of the PTWv lateral dynamics.

\section{ACKNOWLEDGEMENT}

This work is supported by National Agency of Research in France, under the framework VIROLO++ ANR-15-CE220008 : https://anr.fr/Projet-ANR-15-CE22-0008.

\section{APPENDIX}

\begin{tabular}{llll}
\hline$M=303.31$ & & & \\
$e_{34}=6.32$ & $e_{35}=171.38$ & $e_{36}=0.187$ & $e_{44}=34.73$ \\
$e_{45}=1.97$ & $e_{46}=0.66$ & $e_{55}=118.02$ & $e_{56}=0.383$ \\
$e_{66}=0.614$ & & & \\
$\bar{a}_{34}=-303.31 v_{x}$ & $\bar{a}_{44}=-6.319 v_{x}$ & $\bar{a}_{45}=-3.665 v_{x}$ & $\bar{a}_{46}=0.682 v_{x}$ \\
$\bar{a}_{47}=0.856$ & $\bar{a}_{48}=-0.624$ & $\bar{a}_{51}=1681$ & $\bar{a}_{52}=164.34$ \\
$\bar{a}_{54}=-175.048 v_{x}$ & $\bar{a}_{56}=-1.4622 v_{x}$ & $\bar{a}_{61}=164.34$ & $\bar{a}_{62}=69.45$ \\
$\bar{a}_{64}=-0.8685 v_{x}$ & $\bar{a}_{65}=1.47 v_{x}$ & $\bar{a}_{66}=-12.67$ & $\bar{a}_{67}=-0.0894$ \\
$\bar{a}_{71}=-5319 v_{x}$ & $\bar{a}_{72}=104503 v_{x}$ & $\bar{a}_{73}=-112430$ & $\bar{a}_{740}=-84997$ \\
$\bar{a}_{76}=10051$ & $\bar{a}_{77}=-5 v_{x}$ & $\bar{a}_{81}=3221.8 v_{x}$ & $\bar{a}_{83}=-100890$ \\
$\bar{a}_{84}=79098 v_{x}$ & $\bar{a}_{88}=-5 v_{x}$ & & \\
\hline
\end{tabular}

$$
\bar{A}\left(v_{x}\right)=\left[\begin{array}{cccccccc}
0 & 0 & 0 & 0 & 1 & 0 & 0 & 0 \\
0 & 0 & 0 & 0 & 0 & 1 & 0 & 0 \\
0 & 0 & 0 & a_{34} & 0 & 0 & 1 & 1 \\
0 & 0 & 0 & a_{44} & a_{45} & a_{46} & a_{47} & a_{48} \\
a_{51} & a_{52} & 0 & a_{54} & 0 & a_{56} & 0 & 0 \\
a_{61} & a_{62} & 0 & a_{64} & a_{65} & a_{66} & a_{67} & 0 \\
a_{71} & a_{72} & a_{73} & a_{74} & 0 & a_{76} & a_{770} & 0 \\
a_{81} & 0 & a_{83} & a_{84} & 0 & 0 & 0 & a_{88}
\end{array}\right]
$$

\section{REFERENCES}

[1] E. Flanigan, K. Blizzard, A. Rivadeneyra, R. Campbell, and C. Systematics, "Motorcycle safety and intelligent transportation systems gap analysis, final report," United States. Joint Program Office for Intelligent Transportation Systems, Tech. Rep., 2018.

[2] M. E.-H. Dabladji, D. Ichalal, H. Arioui, and S. Mammar, "Unknowninput observer design for motorcycle lateral dynamics: Ts approach," Control Engineering Practice, vol. 54, pp. 12-26, 2016.

[3] L. Nehaoua, D. Ichalal, H. Arioui, J. Davila, S. Mammar, and L. M. Fridman, "An unknown-input hosm approach to estimate lean and steering motorcycle dynamics," IEEE Transactions on Vehicular Technology, vol. 63, no. 7, pp. 3116-3127, 2014.

[4] D. Ichalal, H. Dabladji, H. Arioui, and S. Mammar, "Observer design for motorcycle lean and steering dynamics estimation: a takagi-sugeno approach," in American Control Conference (ACC), 2013.

[5] I. Boniolo, S. M. Savaresi, and M. Tanelli, "Lean angle estimation in two-wheeled vehicles with a reduced sensor configuration," in Circuits and Systems (ISCAS), 2012 IEEE International Symposium on. IEEE, 2012, pp. 2573-2576.

[6] M. Fouka, L. Nehaoua, M. Dabladji, H. Arioui, and S. Mammar, "Adaptive observer for motorcycle state estimation and tire cornering stiffness identification," in 2018 IEEE Conference on Decision and Control (CDC). IEEE, 2018, pp. 3018-3024.

[7] A. Teerhuis and S. Jansen, "Motorcycle state estimation for lateral dynamics," Vehicle System Dynamics, vol. 50, no. 8, pp. 1261-1276, 2012.

[8] P.-M. Damon, D. Ichalal, L. Nehaoua, and H. Arioui, "Lateral \& steering dynamics estimation for single track vehicle: Experimental tests," IFACPapersOnLine, vol. 50, no. 1, pp. 3400-3405, 2017.

[9] R. S. Sharp, "The stability and control of motorcycles," Journal of mechanical engineering science, vol. 13, no. 5, pp. 316-329, 1971.

[10] A. Shabana, Dynamics of Multibody Systems. Cambridge University Press, 2013

[11] K. Tanaka and H. O. Wang, Fuzzy control systems design and analysis: a linear matrix inequality approach. John Wiley \& Sons, 2004.

[12] M. Dabladji, D. Ichalal, H. Arioui, and S. Mammar, "Unknown-input observer design for motorcycle lateral dynamics: Ts approach," Control Engineering Practice, vol. 54, pp. 12-26, 2016.

[13] M. Lazar, D. M. De La Peña, W. Heemels, and T. Alamo, "On input-tostate stability of min-max nonlinear model predictive control," Systems \& Control Letters, vol. 57, no. 1, pp. 39-48, 2008.

[14] S. Boyd, L. El Ghaoui, E. Feron, and V. Balakrishnan, Linear Matrix Inequalities in System and Control Theory, P. SIAM, Ed. SIAM ed., 1994. 